\title{
Synthesis and manufacture of photocrosslinkable poly(caprolactone)-based three-dimensional scaffolds for tissue engineering applications
}

\author{
Nathan Castro ${ }^{1}$, Paul Goldstein ${ }^{1}$, Malcolm Norman Cooke ${ }^{2}$ \\ ${ }^{1}$ Department of Biological Sciences, University of Texas at El Paso, USA; \\ ${ }^{2}$ Mechanical and Aerospace Engineering Department, Case Western Reserve University, Cleveland, USA. \\ Email: pgoldste@utep.edu
}

Received 5 April 2011; revised 23 May 2011; accepted 28 May 2011.

\begin{abstract}
It is known that the body can efficiently repair hard tissue (bone) micro fractures by suturing the defect through the deposition of minerals resulting in an area that is stronger post-injury. Larger defects, however, generally cause more trouble since the body is incapable of repairing them. Bone defects can occur as a result of congenital abnormalities, trauma, or disease. Traditional methods for addressing these defects have involved the use of acellular cadaverous bone or autologous bone. Both contain innate problems associated with them; the former method can result in disease transmission, as well as very low integration with the host due to the lack of viable cells while the latter is associated with two surgical sites and morbidity at the donor site. Alternative methods have been developed, but no method has yet provided a satisfactory solution. As a result, researchers and the medical community are turning toward the promising fields of biomaterial development and tissue engineering to develop new materials and methods of bone regeneration. In this work, a design of experiments (DOE) approach was performed to render commercially available biodegradable polymers (Poly(caprolactone)-diol/triol) photocrosslinkable and resultantly manufacturable using stereolithography (SL), a rapid prototyping technology. To perform the investigations, a commercial SL system (Viper HA, 3D Systems, Valencia, CA) equipped with a solid state laser system (355 $\mathrm{nm}$ wavelength) was used to manufacture synthesized poly(caprolactone) trifumarate (PCLtF) 3D porous constructs. Results of the work conducted produced constructs which provided promising chemical and biological results for the intended application.
\end{abstract}

Keywords: Tissue Engineering; Poly (Carpolactone) Based Scaffolds; Bone Regeneration

\section{INTRODUCTION}

Recent advancements in the area of tissue engineering and the development of porous three-dimensional (3D) implantable scaffolds for tissue regeneration have placed a greater demand on the need for novel biomaterials which exhibit more desirable properties, as well as lend themselves to be modified for a specific application. Traditional methodologies such as salt/porogen leaching have been extensively studied and employed in the "manufacture" of porous scaffolds [1], but several limitations exist; namely, lack of control of uniform pore dispersion, pore geometry, pore size, and interconnectivity all of which are necessary for successful integration of the 3D scaffold with the host [2].

Rapid prototyping (RP) technologies provide a means of addressing the aforementioned limitations of traditional methods through the development of novel biomaterials tailored to a specific RP technology resulting in constructs with desirable and designable properties necessary for their application. One such RP technology that is currently being investigated is stereolithography (SL).

Stereolithography is an RP technology capable of producing highly accurate 3D parts and currently used in a variety of biomedical applications, inparticularm it can be applied to manufacture 3D porous scaffolds for tissue engineering applications. Parts are created by, polymerizing, or photocrosslinking, a resin with an ultraviolet (UV) laser that cures/solidifies the resin in an additive layer-by-layer process until the part is completed. The scientific community has shown signs of distancing themselves from this particular technology, however, no other technology has shown the resolution, accuracy and control of 3D internal and external architecture that can be achieved by SL.

The development of novel polymeric biomaterials that can be manufactured using RP offers engineering ad- 
vantages, including enhanced biocompatibility, biodegradability, and bioactivity. Current research related to polymeric biomaterials involves studies of ways in which these materials can be modified to making them more suitable for specific applications. For example, to address the repair of hard tissue defects $[3,4]$ one such modification could be the addition of hydroxyapatite nanocomposites to the native SL resin. Physically altering the chemical composition of the polymer is another modification utilized in the SL process for the purpose of improving manufacturability. Research and development in these areas are likely to become even more important as technology changes and the biomedical industry continues to demand new materials with improved properties to address specific patient needs.

The approach taken to chemically alter the molecular composition of polymeric biomaterials is critical as much of the physical and mechanical properties of the synthesized material are predicated on the chemical composition of the polymer. With regards to polymeric biomaterials, one key determinant of mechanical properties is molecular weight with stiffness increasing as a function of molecular weight. In addition, solubility decreases with increasing molecular weight, therefore chemical modification of polymeric biomaterials must be planned according to the RP technology to be used. Low molecular weight materials are most suited for manufacture via SL due to the fact that the SL process manufactures from a photocrosslinkable liquid resin. For this reason, it is key to render the candidate material photocrosslinkable while maintaining a relatively low molecular weight.

In addition to manufacturability; biocompatibility, degradability and bioactivity are properties that can be addressed via chemical modification through the incorporation of functional side-/endgroups to the polymeric backbone. Biocompatibility is an important characteristic for successful implantation of 3D TE constructs. It is nearly impossible to introduce a foreign body/material to the physiological system without eliciting a host response, but through chemistry the severity of host response can be controlled through the encapsulation or adsorption of proteins, growth factors, or pharmaceuticals [5]. Degradability and bioactivity are of equal concern and are critical components that are currently being investigated with regards to implantable polymeric biomaterials [6]. Modes of degradation (bulk or surface) and degradation rates can be controlled by altering the molecular weight of the synthesized polymer and can be tailored to the end application.

The current study supports translational biomedical related research in which a proven biocompatible material, poly(caprolactone), has been rendered more clini- cally relevant by tailoring and functionalizing the material for use in a specific rapid prototyping technology for the manufacture of customizable implants. The clinical benefits are numerous and widespread to include the elimination and prevention of additional surgery, better host tissue integration, and fewer complications due to infection by the incorporation of bioactive factors.

\section{MATERIALS AND METHODS}

\subsection{Synthesis of PCLF and PCLtF}

Poly (caprolactone) fumarate (PCLF) and Poly (caprolactone) trifumarate (PCLtF) were synthesized via a condensation polymerization reaction used for the synthesis of poly(propylene fumarate) [7] (Figure 1). All reagents were obtained from Sigma-Aldrich (Sigma -Aldrich, St. Louis, MO 63103). Diethyl fumarate (DEF) and precursor Poly(caprolactone) (PCL) derivatives used in these experiments are currently approved by the United States Food and Drug Administration as biocompatible materials, additionally PCL is marketed as a biodegradable polymer. No purification of reagent materials was conducted. The current synthesis methodology was developed to address existing shortcomings as described by Wang, et al. [8] with respect to color and UV photocrosslinkability, as well as solubility; namely the use of harsh organic solvents due to the insoluble nature of Poly(caprolactone) at high molecular weights $(>2,000$ Daltons).

The glassware setup for the synthesis of each respective material consisted of a three-neck round bottom flask where all reagents were added. The flask was seated in an oil bath set at $100^{\circ} \mathrm{C}$ initially and left for 1 hour. The oil temperature was then raised at a rate of $10^{\circ} \mathrm{C} /$ hour up to the final reaction temperature $\left(140^{\circ} \mathrm{C}\right)$ An overhead stirrer was used to ensure adequate mixture of reagents and is set at $300 \mathrm{rpm}$. A key component to the success of the reaction(s) was a constant vapor temperature $\left(\sim 70^{\circ} \mathrm{C}\right)$ which was measured by a claisen adapter and thermometer. The boiling point of ethanol is $70^{\circ} \mathrm{C}$. A very low flow rate $(<0.012 \mathrm{psi})$ of nitrogen gas $\left(\mathrm{N}_{2(\mathrm{~g})}\right)$ was fed into the system to prevent oxidation of the polymer and the ethanol vapor was fed into a condenser which was connected to a cooling system of chilled water. The flow rate was monitored by a bubble system and the distilled ethanol was collected and measured in a round bottom flask. The reactions were allowed to proceed until $\sim 90 \%$ (weight) of the calculated distillate (ethanol) was collected. Vapor temperature was modulated by adjusting the $\mathrm{N}_{2 \text { (g) }}$ flow rate.

PCLF with a weight-average molecular weight $\left(\mathrm{M}_{\mathrm{w}}\right)$ of 1,475 Da and polydispersity index $\left(M_{w} / M_{n}\right) 1.032$ was synthesized by a transesterification reaction wherein poly (caprolactone) diol $(\mathrm{Mn}=530)$ was reacted with 


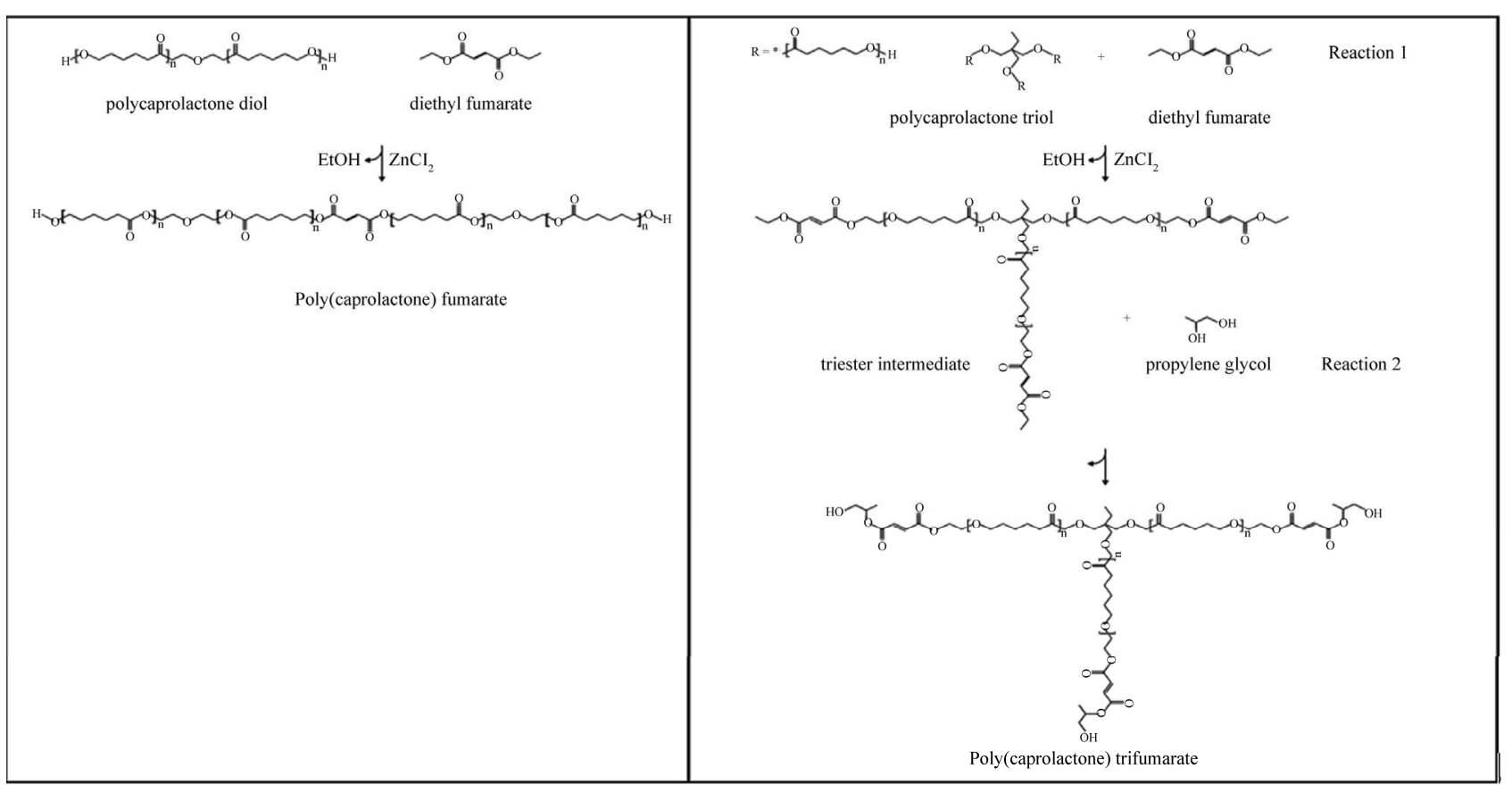

Figure 1. Schematic of (a) Poly(caprolactone) fumate synthesis and (b) Poly(caprolactone) trifumarate synthesis.

diethyl fumarate in the presence of a metal catalyst $\left(\mathrm{ZnCl}_{2}\right)$ and proton scavenger (hydroquinone) (HQ) under an inert atmosphere of nitrogen gas. The amounts of each respective reagent are listed in Table 1.

The quantities used in the synthesis of PCLF were based on stoichiometric ratios of DEF to PCL-diol (2:1) where the fumarate group of DEF is placed in the middle to two PCL polymers such that an hydrolizable $(-\mathrm{OH})$ endgroup of the PCL-diol is retained for biodegradability. The progress of each reaction is determined by the mass of collected distillate (ethanol) which is equivalent to the moles of PCL-diol used in the reaction as PCL is the limiting reagent in this synthesis.

PCLtF with a weight-average molecular weight $\left(\mathrm{M}_{\mathrm{w}}\right)$ of 1,964 Da and polydispersity index $\left(\mathrm{M}_{\mathrm{w}} / \mathrm{M}_{\mathrm{n}}\right) 1.407$ was synthesized by a series of two transesterification reactions wherein poly(caprolactone) triol $(\mathrm{Mn}=900)$ was reacted with diethyl fumarate in the presence of a metal catalyst $\left(\mathrm{ZnCl}_{2}\right)$ and proton scavenger (hydroquinone) (HQ) under an inert atmosphere of nitrogen gas for the purpose of adding the photocrosslinkable alkenecontaining $(\mathrm{C}=\mathrm{C})$ fumarate group. A secondary reaction involving the triester intermediate created from the first reaction and propylene glycol was conducted for the purpose of adding the hydrolizable $(-\mathrm{OH})$ endgroup thus rendering PCLtF biodegradable. The amounts of each respective reagent are listed in Table 2.

Theoretical yields for each of the syntheses were 5:1 and 2:1 for PCLtF (PCLtF:PCL-triol) and PCLF (PCLF: $\mathrm{DEF})$, respectively. Poly(caprolactone) triol was the lim- iting reagent in the synthesis of PCLtF and DEF was the limiting reagent for PCLF synthesis. Actual yields approximated that of the calculated theoretical values.

\subsection{Fourier-Transform Infrared Spectroscopy}

All reagents, synthesized PCLF and PCLtF, as well as the PCLtF triester intermediate were analyzed by Fourier-transform infrared spectroscopy (FT-IR) (Perkin Elmer Spectrum 100 FT-IR Spectrometer, Perkin Elmer, Waltham, MA 02451) using a salt plate. Sodium chloride is "invisible" at the infrared range of the spectrum. The salt plate was washed with acetone prior to each analysis. FT-IR analysis exploits the oscillating frequencies of bonded molecules to characterize functional groups based on mass differences; the oscillating frequencies of the molecule provide a fingerprint of the molecule.

\subsection{Molecular Weight/Polydispersity Determination}

Molecular weight analysis and polydispersity index determination were conducted by gel permeation chromatography (GPC) (Viscotek GPCMax/TDA, Malvern Instruments Ltd., Worcestershire, United Kingdom). GPC is a size-exclusion based chromatographic technique wherein the dissolved analyte is injected in-stream with the mobile phase separated by an air bubble. The refractive index of the analyte was compared with the refractive index of the solvent (mobile phase) upon passage through a porous silica column where larger particles 
Table 1. Quantities of each respective reagent used in the synthesis of PCLF.

\begin{tabular}{cccc}
\hline & Poly(caprolactone) fumarate synthesis & \\
\hline Reagent & Mol Ratio & Formula Weight $\left(\mathrm{g} \cdot \mathrm{mol}^{-1}\right)$ & Mass $(\mathrm{g})$ \\
\hline Diethyl fumarate & 1 & 172.18 & 172.18 \\
Poly(caprolactone) diol & 2 & 114.1 & 228.2 \\
Hydroquinone (HQ) & 0.01 & 110.11 & 1.1011 \\
Zinc Chloride (ZnCl $\left.{ }_{2}\right)$ & 0.002 & 136.28 & 0.2726 \\
Ethanol (Distillate) & 2 & 46 & 92 \\
\hline
\end{tabular}

Table 2. Quantities of each respective reagent used in the synthesis of PCLtF.

\begin{tabular}{cccc}
\hline & Poly(caprolactone) trifumarate synthesis & & \\
\hline Reagent & Mol Ratio & ${\text { Formula Weight }\left(\mathrm{g} \cdot \mathrm{mol}^{-1} \text { ) }\right.}$ & Mass (g) \\
\hline Diethyl fumarate & 3 & 172.18 & 516.54 \\
Poly(caprolactone) diol & 1 & 114.1 & 114.1 \\
Hydroquinone (HQ) & 0.01 & 110.11 & 1.1011 \\
Zinc Chloride (ZnCl ${ }_{2}$ ) & 0.002 & 136.28 & 0.2726 \\
Propylene glycol & 3 & 76.1 & 228.3 \\
Ethanol (Distillate) & 6 & 46 & 276 \\
\hline
\end{tabular}

matriculate first through the column and smaller molecules are impeded by the porous silica. Polystyrene (PS) standards of varying molecular weights $(2,000-25,000$ Daltons) were analyzed for calibration of the instrument. PS standard solutions $(<25 \mathrm{mg} / \mathrm{mL}$ ) used in the analysis were unfiltered due to the relative purity of the sample. Tetrahydrofuran was used as the organic mobile phase and dissolved PCLF/PCLtF samples were diluted in varying concentrations $(<250 \mathrm{mg} / \mathrm{mL})$ and filtered through a $0.2 \mu \mathrm{m}$ syringe filter prior to analysis.

\subsection{Results and Discussion}

Synthesized Poly(caprolactone) fumarate (PCLF) and Poly(caprolactone) trifumarate (PCLtF) as shown in Figure 1 were chemically characterized using Fouriertransform infrared spectroscopy (Perkin Elmer Spectrum 100 FT-IR Spectrometer, Perkin Elmer, Waltham, MA). Molecular weight and polydispersity index determination were conducted by gel permeation chromatography (GPC) (Viscotek GPCMax/TDA, Malvern Instruments Ltd., Worcestershire, United Kingdom).

\subsection{Fourier-Transform Infrared (FT-IR) Spectroscopy Results and Discussion}

\subsubsection{Poly(caprolactone) Fumarate}

Poly(caprolactone) fumarate was synthesized by the same methodology used in synthesizing PCLtF. Two PCLF derivatives were synthesized and analyzed by FT-IR. The purpose of the experiment was to incorporate a photocrosslinkable alkene $(\mathrm{C}=\mathrm{C})$ between two PCL molecules. Unlike the two-step PCLtF reaction, only one reaction was conducted as one of the primary alcohols present in the PCL-diol molecule was retained for biodegradability. PCL-diol is an aliphatic polyester so named due to the two primary alcohol endgroups containing hydroxyl endgroups $(-\mathrm{OH})$ present. Two PCLdiols of nominal weight $\left(\mathrm{M}_{\mathrm{n}}\right) 530 \mathrm{Da}$ and $1250 \mathrm{Da}$ were used, respectively. PCL530 exhibited a less viscose appearance as opposed to PCL1250 due to the lower molecular weight.

FT-IR spectra of DEF, PCL530, PCL1250, PCLF530, and PCLF1250 are shown in Figure 2. Similar infrared

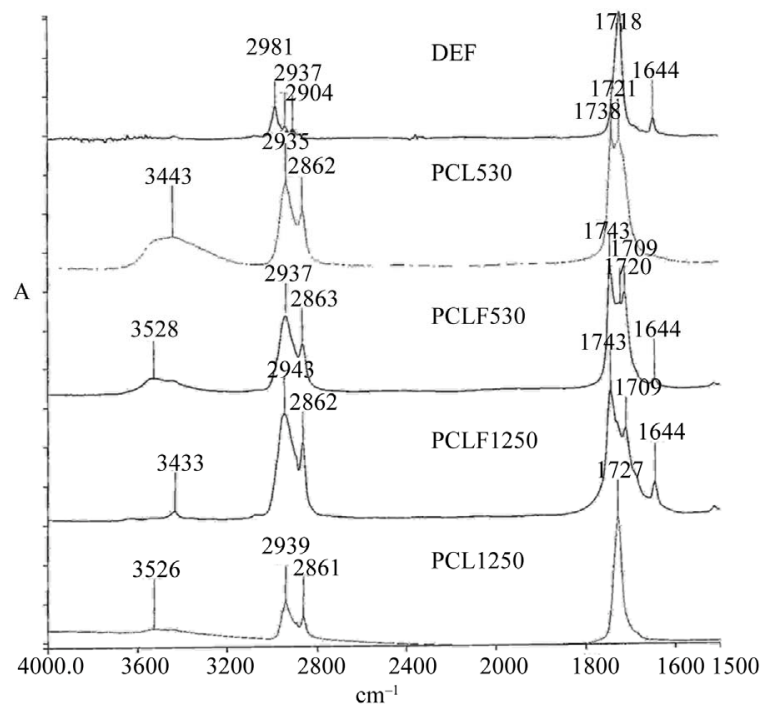

Figure 2. FT-IR spectra of DEF, PCL and synthesized PCLF. 
absorption $\sim 3500 \mathrm{~cm}^{-1}$ and $\sim 1735 \mathrm{~cm}^{-1}$ were present in both PCL-diols used in the synthesis of PCLF. As in the case of PCLtF, the primary peak of interest with regards to DEF was that of the crosslinkable alkene $(C=C)$, which is typically seen $\sim 1650 \mathrm{~cm}^{-1}$. PCLF530/1250 was synthesized through a one-pot transesterification reaction. The reaction was performed for the purpose of adding flexible hydrolysable PCL groups to a center alkene. The product yielded a photocrosslinkable PCL. FT-IR analysis of synthesized PCLFs revealed the presence of the alkene and hydroxyl peaks.

\subsubsection{Poly(caprolactone) Trifumarate}

Poly(caprolactone) trifumarate was synthesized via a condensation polymerization reaction. The purpose of the experiment was to functionalize low molecular weight poly(caprolactone) $(<1,250 \mathrm{Da}$ ) by incorporating photocrosslinkable alkene endgroups to the PCL molecule. In addition to rendering the material crosslinkable, a secondary reaction was conducted to render the end product biodegradable via hydrolysis.

FT-IR spectra of the two main reagents (Diethyl fumarate (DEF) and Poly(caprolactone) triol (PCL-triol) are shown in Figure 3. PCL-triol is so named due to the presence of three functional primary alcohols containing hydroxyl endgroups (-OH). Infrared absorption by $-\mathrm{OH}$ typically occurs $\sim 3500 \mathrm{~cm}^{-1}$ [9] as indicated in Figure 3 . A secondary PCL-triol peak of interest was that of the carbonyl $(\mathrm{C}=\mathrm{O})$ which absorbed at $\sim 1735 \mathrm{~cm}^{-1}$. The primary peak of interest with regards to DEF was the crosslinkable alkene $(C=C)$ which was seen $\sim 1650 \mathrm{~cm}^{-1}$.

PCLtF was synthesized through a series of two reactions. The first reaction was performed for the purpose of adding the crosslinkable alkene to the PCL branches. The product of the first reaction was a PCL-triester intermediate. FT-IR analysis of the intermediate illustrated a diminished hydroxyl peak and a detectable alkene peak. The by-product of the both reactions was ethanol, therefore the diminishing hydroxyl peak corresponded to the distilled ethanol. The PCL-triester intermediate was the limiting reagent for the second reaction.

The second PCLtF synthesis reaction involved the use of the triester intermediate from the first reaction in addition to a highly used FDA approved biocompatible material, propylene glycol (PG) (Figure 4). FT-IR spectral analysis revealed the presence of the hydroxyl group present in the PG molecule. PG was reacted with the triester intermediate through a transesterification reaction for the purpose of adding the hydrolysable hydroxyl functional group thus rendering PCLtF biodegradable through hydrolysis. FT-IR spectra of PCLtF revealed the successful incorporation of the alkene and hydroxyl functional endgroup.

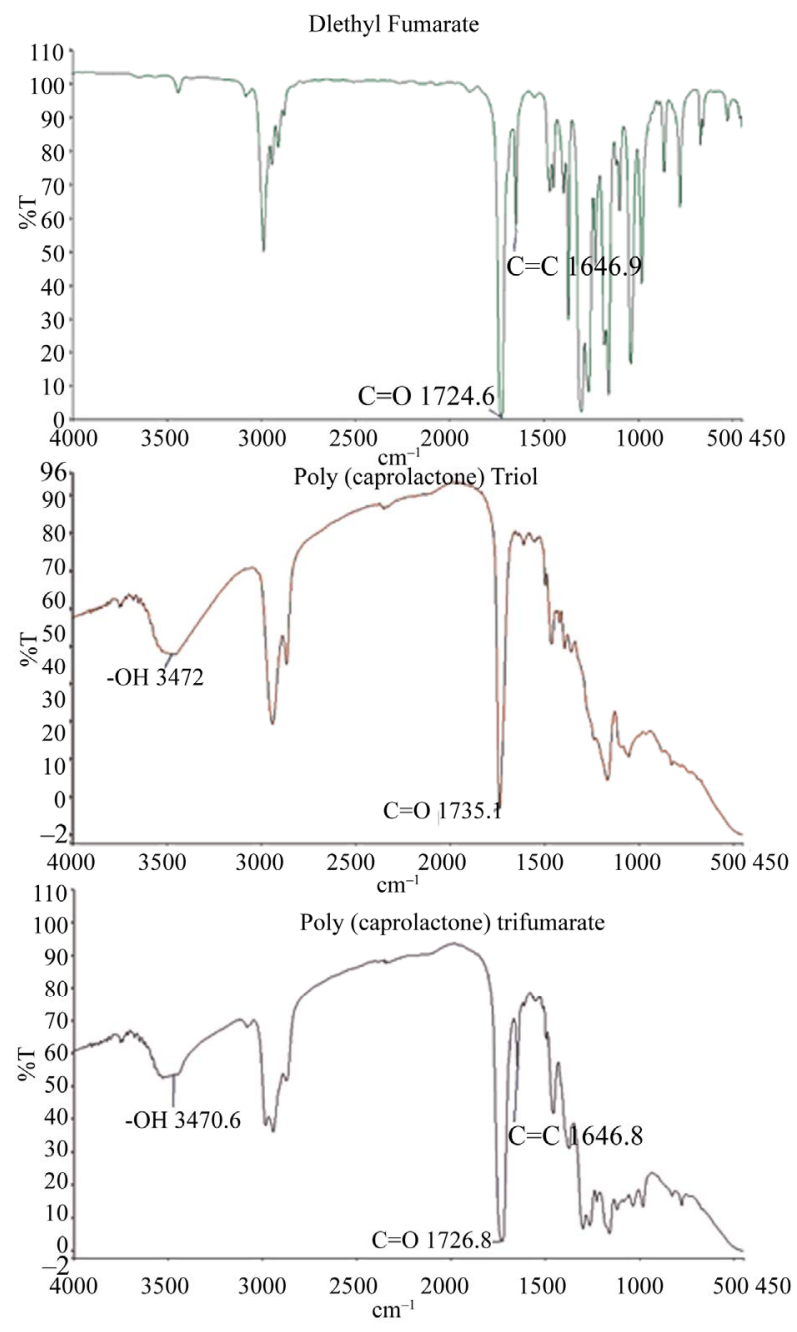

Figure 3. FT-IR spectra of PCLtF synthesis reaction 1.

\subsection{Molecular Weight/Polydispersity Results and Discussion}

\subsubsection{Poly(caprolactone) Fumarate}

GPC analysis of synthesized PCLF (Figure 5) revealed a molecular weight of 1,475 Daltons with a corresponding PI of 1.032 (Figure 6).

\subsubsection{Poly(caprolactone) Trifumarate}

GPC analysis of synthesized PCLtF (Figure 7) revealed a molecular weight of 1,964 Daltons with a corresponding PI of 1.407 (Figure 8).

Molecular weight and polydispersity indices affect, to a large extent, many physical and mechanical properties to include: degradation rate, mode of degradation, manufacturability, and solubility. Therefore, it is imperative to functionalize PCL while maintaining a manageable molecular weight. The results presented in Figures 5-8 illustrate molecular weights below the desired maximum of 2,000 Daltons and PI less than 2.0. A low PI indicates 


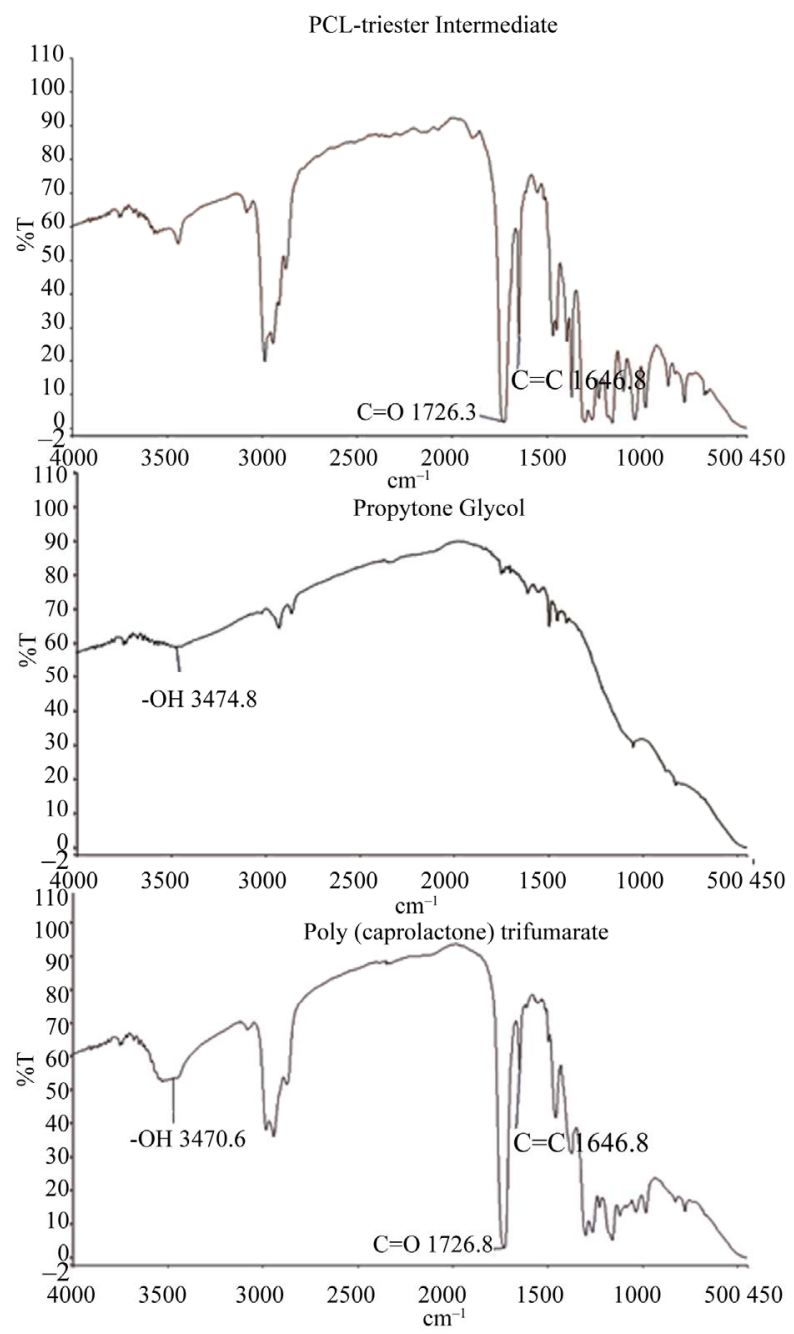

Figure 4. FT-IR spectra of PCLtF synthesis reaction 2.

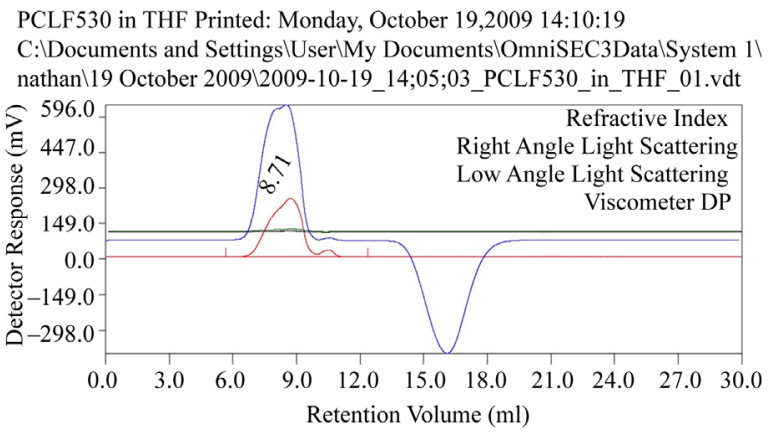

Figure 5. Chromatogram of synthesized PCLF.

a more uniform chain length which leads to more uniform degradability while low molecular weight materials degrade at a faster rate.

We have demonstrated that commercially available low molecular weight PCL can be functionalized and rendered photocrosslinkable by adapting a polyconden sation reaction using FDA approved reagents. The meth- odology employed lends itself to be extended to other primary alcohol containing biodegradable reagents, thus, broadening the range of materials that can be rendered photocrosslinkable. Also, the elimination of harsh solvents is an additional benefit which has implications in terms of cell adhesion and biocompatibility.

Studies utilizing Poly(caprolactone)-based materials have primarily, if not entirely, focused on hard tissue regeneration, thus, limiting it's applicability in terms of regenerative medicine. Studies have indicated that branched molecules are more readily soluble than their ali-

\begin{tabular}{|l|r|}
\hline Peak RV - (ml) & 8.713 \\
\hline Mn - (Daltons) & 1,429 \\
\hline Mw - (Daltons) & 1,475 \\
\hline Mz - (Daltons) & 1,576 \\
\hline Mp - (Daltons) & 1,432 \\
\hline Mw/Mn & 1.032 \\
\hline Percent Above Mw: 0 & 0.000 \\
\hline Percent Below Mw: 0 & 83.556 \\
\hline IV - (dl/g) & 0.0461 \\
\hline Rh - (nm) & 1.009 \\
\hline Rg - (nm) & No Calc \\
\hline Wt Fr (Peak) & 1.000 \\
\hline Mark-Houwink a & 1.517 \\
\hline Mark-Houwink logK & -6.121 \\
\hline Branches & 0.000 \\
\hline Branch Freq. & 0.000 \\
\hline RI Area - (mvmml) & 442.98 \\
\hline UV Area - (mvmml) & 0.00 \\
\hline RALS Area - (mvml) & 16.70 \\
\hline LALS Area - (mvml) & 9.73 \\
\hline DP Area - (mvml) & 1085.59 \\
\hline
\end{tabular}

Figure 6. Gel permeation chromatography results of synthesized PCLF with highlighted molecular weight and polydispersity index (Mw/Mn).

PCLtF in THF Printed: Monday, October 05,2009 13:48:02 C:LDocuments and SettingsiUserlMy Documents\OmniSEC3Data\System $1 \backslash$ nathan $\backslash 25$ Sep 09\2009-09-25 13;19;50 PCLtF in THF 01.vdt

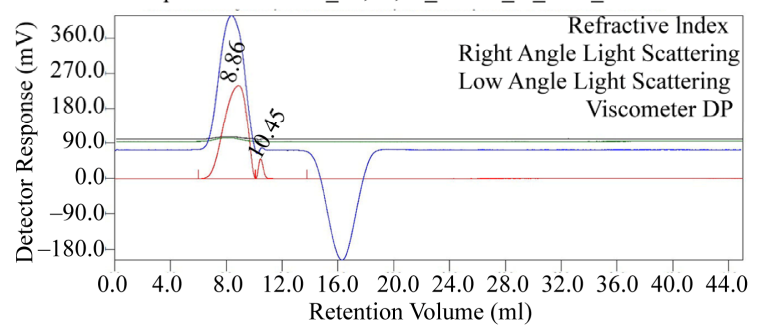

Figure 7. Chromatogram of synthesized PCLtF. 


\begin{tabular}{|l|r|}
\hline Peak RV - (ml) & 8.863 \\
\hline Mn - (Daltons) & 1,396 \\
\hline Mw - (Daltons) & 1,964 \\
\hline $\mathrm{Mz}$ - (Daltons) & 3,229 \\
\hline $\mathrm{Mp}$ - (Daltons) & 1,302 \\
\hline Mw/Mn & 1.407 \\
\hline Percent Above Mw: 0 & 0.000 \\
\hline Percent Below Mw: 0 & 6.425 \\
\hline IV - (dl/g) & 0.0339 \\
\hline Rh - (nm) & 0.983 \\
\hline Rg - (nm) & No Calc \\
\hline Wt Fr (Peak) & 0.958 \\
\hline Mark-Houwink a & 0.544 \\
\hline Mark-Houwink logK & -3.237 \\
\hline Branches & 0.000 \\
\hline Branch Freq. & 0.000 \\
\hline RI Area - (mvml) & 450.55 \\
\hline UV Area - (mvml) & 0.00 \\
\hline RALS Area - (mvml) & 21.10 \\
\hline LALS Area - (mvml) & 12.89 \\
\hline DP Area - (mvml) & 691.71 \\
\hline
\end{tabular}

Figure 8. Gel permeation chromatography results of synthesized PCLF with highlighted molecular weight and polydispersity index (Mw/Mn).

phatic counterparts, therefore, PCLtF will be the focus of crosslinking studies. In addition to an increased miscibility, the branched molecule provided a greater crosslinking density, such that the structural integrity of the crosslinked material could be increased. The inherent miscibility of the synthesized PCL derivatives with the precursor reagent, diethyl fumarate (DEF), as well as the low molecular weight, lend to the use and manufacture of this synthesized PCLF/PCLtF.

\section{ACKNOWLEDGEMENTS}

This publication was made possible in part by Grants \# $\underline{\text { G12RR008124 }}$ to the Border Biomedical Research Center (BBRC)/University of Texas at El Paso from the National Center for Research Resources (NCRR)/
NIH and NIH/NIDCR 5SC2DE019725-02. The contents of this publication are solely the responsibility of the authors and do not necessarily represent the official views of NIH or NIEHS.

\section{REFERENCES}

[1] Smith, I.O., Liu, X.H., Smith, L.A. and Maet, P. (2009) Nanostructured polymer scaffolds for tissue engineering and regenerative medicine. Wiley Interdisciplinary Reviews: Nanomedicine and Nanobiotechnology, 1, 226236. doi:10.1002/wnan.26

[2] Karageorgiou, V. and Kaplan, D. (2005) Porosity of 3D biornaterial scaffolds and osteogenesis. Biomaterials, 26, 5474-5491. doi:10.1016/j.biomaterials.2005.02.002

[3] Baji, A., Wong, S., Srivatsan, T., Njus, G. and Mathur, G. (2006) Processing methodologies for polycaprolactonehydroxyapatite composites: A review. Materials and Manufacturing Processes, 21, 211-218.

doi:10.1081/AMP-200068681

[4] Sopyan, I., Ramesh, I. and Khalidet, K. (2007) Porous hydroxyapatite for artificial bone applications. Science and Technology of Advanced Materials, 8, 116-123. doi:10.1016/j.stam.2006.11.017

[5] Angel, M.J., Sgaglione, N. and Grande, D. (2006) Clinical applications of bioactive factors in sports medicine current concepts and future trends. Sports Medicine and Arthroscopy Review, 14, 138-145. doi:10.1097/00132585-200609000-00005

[6] Golebiewski, J., Gibas, E. and Malinowski, R. (2008) Selected biodegradable polymers - preparation, properties, applications. Polimery, 53, 799-807.

[7] Shung, A.K., Timmer, M., Seongbong, J., Engel, P. and Mikos, A. (2002) Kinetics of poly(propylene fumarate) synthesis by step polymerization of diethyl fumarate and propylene glycol using zinc chloride as a catalyst. Journal of Biomaterials Science-Polymer Edition, 13, 95-108. doi:10.1163/156856202753525963

[8] Wang, S.F., Kempen, D., Simha N., Lewis J., Windebank A., Yaszemski, M. and Lu, L. (2008) Photo-cross-linked hybrid polymer networks consisting of poly (propylene fumarate) and poly (caprolactone fumarate): Controlled physical properties and regulated bone and nerve cell responses. Biomacromolecules, 9, 1229-1241. doi:10.1021/bm7012313

[9] Pretsh, E., Buhlmann, P. and Affolter, C. (2000) Structure determination of organic compounds. 3rd Edition, Springer, Heidelberg, 421. 